\title{
Specific eye fundus lesions in type II membranoproliferative glomerulonephritis
}

\author{
Anita Leys', Willem Proesmans², Rita Van Damme-Lombaerts ${ }^{2}$, and Boudewijn Van Damme 3 \\ Departments of ${ }^{1}$ Ophthalmology, ${ }^{2}$ Paediatrics, and ${ }^{3}$ Pathology, University Hospital, Gasthuisberg, Herestraat, B-3000 Leuven, Belgium 1
}

Received June 15, 1990; received in revised form October 12, 1990; accepted November 16, 1990

Due to a technical error, the following line drawings were produced wrong-reading on page 190 of volume 5 , issue $2,1991$.

Please excuse this mistake.
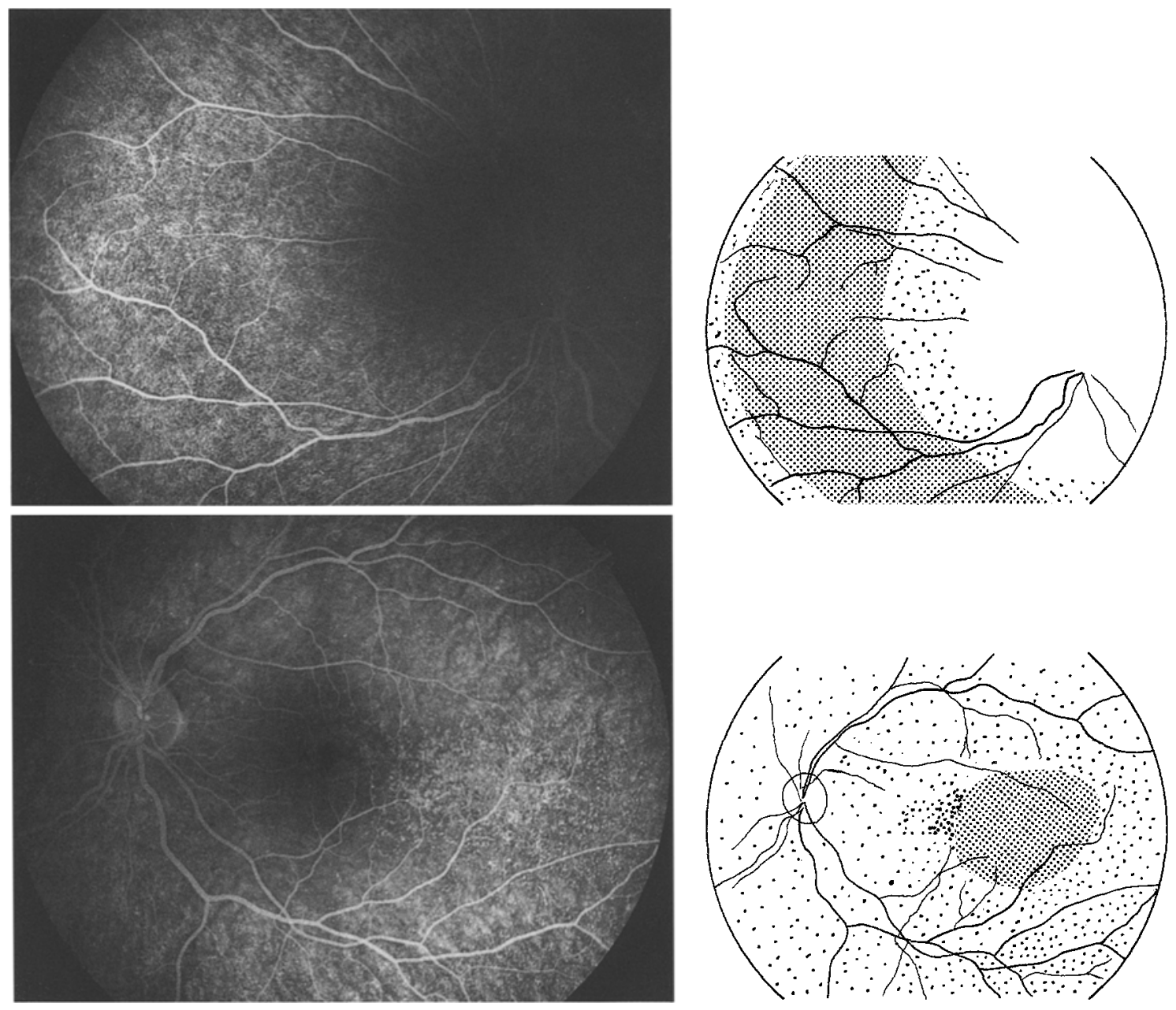

Fig. 1. Patient 1. Fluorescein angiogram of the left eye, nasal (top) and macular view (bottom). Basal laminar drusen are randomly scattered, but are more closely arranged in the shaded parts 